\title{
Kassel ohne Athen: \\ Die documenta 14 zerbricht an ihrer Ortslosigkeit
}

Frank Eckardt

Als eine ,Übung der Zerbrechlichkeit‘ hat sich die diesjährige Documenta präsentiert, zumindest aus ihrer eigenen Sichtweise. An jeder Eingangsstelle wurde dieser Slogan den Besucher_innen aus Lautsprechern entgegengeflüstert. Als weiteres Narrativ hatte die Kunstausstellung die Losung ,Von Athen lernen“ ausgegeben - ein Satz, der schon weit im Vorfeld für viel Diskussion gesorgt hatte und der nirgendwo im eigentlichen Sinne erklärt wurde. Formuliert wurde er auf dem Höhepunkt der Schuldenkrise Griechenlands und von vielen als eine Art von Solidaritätserklärung verstanden. Was aber genau von Athen gelernt werden sollte, haben die Kurator_innen nie ausgeführt. Von Beginn an war dadurch ein Vermittlungsproblem gegenüber der lokalen Bevölkerung in Kassel entstanden, die die Aufteilung auf zwei Standorte als einen Verlust betrachtete. Insbesondere die lokale CDU lehnte diesen Ansatz weitgehend ab. Die Befürchtung war entstanden, dass sich einerseits die Documenta als eine Art globale Wanderausstellung vollkommen von Kassel entfremden würde und dass sie sich andererseits an einer solchen doppelten Ausrichtung verheben würde. Letzteres hat sich finanziell auf jeden Fall bewahrheitet und somit zugleich auch die inhaltliche Kritik, mit der documenta 14 werde die Ausstellung ortsfremd, weiter befeuert. Positive Interpretator_innen, zu denen der damalige Oberbürgermeister Bertram Hilgen (SPD) gehörte, unterstrichen die künstlerische Deutungsfreiheit, ohne jedoch unbedingt den damit verbundenen Akt der Solidarisierung mit Griechenland explizit zu unterstützen. Wie ,Von Athen lernen' gemeint sein könnte, blieb nach Auffassung der Ausstellungsmacher_innen den Besucher_innen überlassen. Dabei schien die Verschiebung des Ausstellungsbeginns, mit der die Documenta am Rande Europas und in einem austeritätspolitisch gebeutelten Land ihren Anfang nehmen sollte, die gewollte Perspektivverschiebung zu sein. Das künstlerische Konzept begann dementsprechend mit einer politischen Verortung. Wenn man den Begriff des ,Lernens ${ }^{6}$ - der hier zentral benutzt wurde - weiter thematisiert, dann war dieses Konzept als ein pädagogisch-moralisierendes zu verstehen, womit sich diese Documenta von einem künstlerischem Diskurs abgegrenzt hätte, der sich aus sich selbst heraus legitimiert, und aufjede Selbst-Bezüglichkeit der Kunst verzichten will. 
Stattdessen operierte die Documenta mit einem unklaren, vagen und nichtdiskursiv konstruiertem Narrativ, das sich auch nicht über örtliche Debatten auszeichnete, wie etwa die documenta 10. Eine intellektuelle Auseinandersetzung über das eigene Kunst- und Politikverständnis wurde konzeptionell ausgeschlossen, indem die Performanz und nicht die Auseinandersetzung als Kern der documenta 14 behauptet wurde. So wurden Besucher_innen zu Zuschauer_innen und Zuhörer_innen, die etwas von ,Athen lernen'konnten, denen aber die Gelegenheit des Dialogs nicht eingeräumt wurde. Was von Athen gelernt werden konnte, wurde gezeigt, aber nicht weiterentwickelt, angewandt oder kritisch reflektiert. Die Ausstellung verfolgte damit intrinsisch das Ziel, ein großes Narrativ zu konstruieren, dem man sich anschließen sollte, das sich aber einem nur in der Gesamtschau ergeben konnte und die vielen Einzelkunstwerke belegen sollte. Dieses Narrativ ist die große Erzählung der sozialen Ungerechtigkeit.

Das Narrativ der documenta 14, umstritten und in den Augen vieler Kritiker_innen nicht überzeugend, scheiterte daran, dass es zwar für die neoliberalen Lebensumstände in Athen eine hohe Sensibilität herstellte, diese aber für Kassel vollkommen verweigerte. Allzu deutlich wurde, dass diese Documenta in vieler Hinsicht, anders' war und dies vor allem in ihrer Beziehung zum Ursprungsort der Ausstellung, zu Kassel. Paradoxerweise forderte sie eine Zerbrechlichkeit von den Besucher_innen ein, die sie mit Bezug auf Kassel selbst nicht einüben wollte. Hätte die documenta 14 ihre Sensibilität ins Spiel gebracht, hätte sie ihren gesellschaftskritischen Ausgangspunkt sehr gut vor Ort demonstrieren und eine Art Gleichberechtigung zwischen Athen und Kassel demonstrieren können. Das hat sie aber nicht, maximal rudimentär oder aus großer Distanz getan. Stattdessen hat sie sich weit in die Innenräume der Museen zurückgezogen und sich anhand einer Aneinanderreihung von zahlreichen, zumeist alten Kunstwerken auf ein Narrativ konzentriert, das die lange Geschichte des Unrechts erzählt: vom Kolonialismus, den Verbrechen an Indigenen, den Diktaturen Lateinamerikas, dem deutschen Nationalsozialismus bis zur griechischen Finanzkrise. Der Schwerpunkt lag auf der Erinnerung an vergangenes Unrecht, die Gegenwart vor den Türen der Documenta-Blase blieb fremd.

\section{Tuchfühlung in der Neuen Hauptpost}

Wenn man eine Erschließung der Stadt durch die documenta 14 noch im Ansatz erkennen wollte, dann wohl am ehesten in der eigens für die Ausstellung geöffneten Neuen Hauptpost hinter dem Holländischen Platz. In diesem ehemaligen Verwaltungsgebäude im Stil der brutalistischen Architektur befinden sich heute soziale Einrichtungen wie das Beratungsprojekt für unbegleitete minderjährige Flüchtlinge und die Caritas. Die Kantine und zwei weitere Stockwerke des wuchtigen Gebäudes der 1970er-Jahre sind seit langem leerstehend und kaum im öffentlichen Bewusstsein präsent. Die Documenta hier hinzuverlegen, schien jene Tradition der Kasseler Ausstellung fortzusetzen, sich immer auch mit einem neuen Blick auf Kassel einzubringen. Doch dass dieser Eindruck trog, lernte man schon allzu schnell. In dieser „Neuen neuen Galerie“ titulierten Räumlichkeit wurden immerhin zwei Themen aufgegriffen, die sich mit Kassel beschäftigten, zum 
Beispiel die Geschichte des NSU-Mordes an dem Kasseler Halit Yozgut. Das Künstler-Kollektiv „Forensic Architecture“ hatte mit Ayse Gülec - die sich für die „Freunde von Halit“, der „Initiative 6. April“ und „Kassel postkolonial“ engagiert - einen Workshop beim „FC Bosporus e.V.“ durchgeführt, um die Hintergründe des Mordes aufzuklären. Eine lange Videoreportage war dazu zu sehen. Ayse Gülec ist Sozialarbeiterin im Kulturzentrum „Schlachthof“ und sie war bei der letzten Documenta eine von 40 Vertreter_innen der Stadtgesellschaft, die im Documenta-Beirat mitgearbeitet haben. Einen Beirat der Stadtgesellschaft hatte diese Documenta nicht, der "Schlachthof" wurde als Partner und Veranstaltungsort aufgenommen. Beim traditionellen internationalen Sommerfest sah man Kurator Adam Szymczyk, ansonsten im Grunde in der Stadt unsichtbar, ekstatisch tanzen. Weiterhin wurde eine Fotoausstellung gezeigt, die ebenfalls hauptsächlich ältere Migrant_innen - zufällig auch vom „FC Bosporus“ - darstellte. Ahlam Shiblis wollte mit ihren fotografischen Arbeiten zum Thema „Heimat“ an frühere Projekte in Palästina anknüpfen. Mit „Heimat“ wurden nun die Gastarbeiter_innen der 1950er bis 1970er-Jahre aus Südeuropa und Nordafrika und die Vertriebenen nach dem Zweiten Weltkrieg zusammengeführt.

„Heimat" und die Rekonstruktion der NSU-Morde gingen, das mag man ihnen zugutehalten, zumindest mit Kasseler Themen in Tuchfühlung. Dennoch blieben beide Beiträge auf eine merkwürdige Art der Kasseler Normalität, der gelebten Multikulturalität, fern. War das wirklich alles, was die documenta 14 von einer Stadt wahrnahm, die beruhigend wenig Schwierigkeiten mit dem Zusammenleben von Menschen mit so vielen unterschiedlichen Lebensstilen hat? Der Mord an Halit Yozgut wird seit Jahren in Kassel intensiv diskutiert und hat Untersuchungsausschüsse auf Bundes- und Landesebene beschäftigt. Er war Thema unzähliger Reportagen und vieles mehr. Was hatte die künstlerische Auseinandersetzung dem noch hinzuzufügen? Die Stadtgesellschaft geht damit kreativer um als die Documenta. So zog beispielsweise der Stadtimker Victor Hernández Honig mit seiner „Bienothek“ in das, seit dem Mord verlassene, Internetcafé in der Holländischen Straße ein. Er wollte damit bewusst ein Zeichen dafür setzen, dass sich die Stadt nicht in ihrer Lebendigkeit unterkriegen lässt. So wurde der NSU-Skandal auf dieser Documenta zwar ausgestellt, aber nicht mehr künstlerisch oder kreativ angegangen. Gleiches lässt sich zu Ahlam Siblis Reportage sagen. Die Bezeichnung Reportage - , ich verwende diesen Ausdruck hier, da eine ausführliche Diskussion darüber zu viel Raum einnehmen würde“, so die Künstlerin auf der Documenta-Homepage - sagt im Prinzip schon alles. Hier wurde berichtet und ausgestellt. Hier wurde nicht kommuniziert, interveniert oder dialogisch mit der Kasseler Stadtgesellschaft umgegangen. Man kann das als eindimensionale Kommunikation verstanden wissen, als ein Statement und eine Belehrung. Kann man es eigentlich noch anders auffassen?

\section{Mythos Nordstadt}

Der Parcours der documenta 14 erstreckte sich bis zu den Henschel-Hallen im Nordwesten der Stadt. Dieser Ort wäre eine ideale Räumlichkeit, um sich mit der Frage der Kontinuität der Arbeiter_innengeschichte Kassels auseinanderzusetzen. Die Familie Henschel hat dem industriellen Kassel 
ihren Stempel aufgedrückt. Mit den Henschel-Werken wäre auch die Geschichte der Rüstungsindustrie zu erzählen und der Bogen zur Zerstörung der Stadt durch die Alliierten zu schlagen gewesen. Aber damit würde der Blick wiederum zu verengt. Insbesondere die Fabrikantengemahlin Sophie Henschel hat sich nämlich - zwar auf paternalistische Weise - um die Wohnbedingungen der Arbeiter_innen gekümmert: Noch bis heute bestehen Wohnanlagen unweit des Documenta-Parcours, die ein Verständnis für die Ursprünge einer sozialen Marktwirtschaft vermitteln könnten. Doch daran war der Documenta nicht gelegen. Die Henschel-Hallen wurden stattdessen für gelegentliche Performances genutzt. Die meisten Besucher_innen haben sich hierhin nicht verirrt. Der Anspruch der documenta 14, in die Nordstadt hineinzuwirken, hörte damit geografisch gesehen mit Agnes Denes“ „The Living Pyramid“ im Nordstadtpark auf. Dieses ökologische Monument verschönerte den Park zwar sicherlich. Angelegt direkt hinter dem „Schlachthof“ gehört er aber sozialräumlich zu dem Stadtviertel, das sich über die Mombach- und Gottschalkstraße seit jeher eher der Universität zuordnen lässt, als der Nordstadt. Hier findet seit 30 Jahren mit dem „Café Nordstern“ und der „Unibuch-Buchhandlung“ ein gewisses Straßenleben statt, das in den 1990er-Jahren durch Döner-Imbisse und seit ein paar Jahren durch weitere Restaurants weiter aufblüht. Der Ausbau der Gebäude der „Fakultät Architektur-Stadtplanung-Landschaftsplanung“ an dieser Stelle hat diesen Prozess durch eine visuelle Aufwertung sicherlich noch unterstützt. Es ist deshalb relativ bequem, sich hier als Teil der Nordstadt zu fühlen, während die harte Wirklichkeit sozialer Ausschlüsse erst dort sichtbar und spürbar wird, wo keine Döner an Akademiker_innen verkauft werden, sondern die Kasseler Tafel ihre täglichen Armenspeisungen durchführt. Dazu müsste man nach Nordholland oder zum Fasanenhof gehen, wohin man aber fußläufig wiederum nicht schnell und bequem kommt.

Zu glauben, man sei in der Nordstadt angekommen, wenn man sich einmal an den „Stern“ begibt - also dorthin, wo die Fußgängerzone der Innenstadt aufhört und sich viele türkisch-arabische Cafés und Restaurants befinden -, war eine Illusion, die die Ortsfremdheit der documenta 14 schmerzhaft verdeutlichte. Das wurde anhand der sogenannten Glaspavillons an der KurtSchumacher-Straße überdeutlich. Diese rücken in eigentlich begrüßenswerter Weise das seit einiger Zeit leerstehende ehemalige Hansa-Gebäude ins Bewusstsein. Dennoch war die Wahrnehmung dieses Ortes durch die Documenta schlichtweg falsch und ahistorisch: „Als eine der Hauptverkehrsadern Kassels markiert die Kurt-Schumacher-Straße auch eine Grenze. Geografisch trennt sie das Zentrum von der Nordstadt und bezeichnet so auch eine soziopolitische Demarkation." (Homepage der documenta 14) In Wirklichkeit stellt die Kurt-Schumacher-Straße die Umsetzung des Konzepts der autofreundlichen Stadt durch das Ringstraßenkonzept dar. Sie trennt keineswegs die Innenstadt und die Nordstadt und war auch nicht so intendiert. Im Gegenteil, hinter der Kurt-Schumacher-Straße wurde die Hauptpost und die Universität angesiedelt, die man sicherlich als eine Art Fortsetzung des Stadtzentrums zu verstehen hat. Auch die Behauptung, „die Mitte ist das vergleichsweise homogene wirtschaftliche und kulturelle Zentrum der Stadt“ (ebd.), überrascht. Das Stadtzentrum war bis in die 2000er-Jahre von einem wirtschaftlichen Niedergang gezeichnet und durch Ansiedlungen - wie dem 
Abb. 1 Olu Oguibes' Obelisk auf dem Königsplatz (Eigenes Foto)
Shopping Center DEZ in Niederzwehren - massiv bedroht. Die Innenstadt ist vermutlich mehr als je zuvor von Konkurrenzkämpfen zwischen einheimischen Betrieben und Ketten wie Starbucks und Peek\&Cloppenburg bedroht. Lokale Buchhändler_innen haben ihre Geschäfte zuerst geschlossen, dann Kleidungs- und Schuhverkäufer_innen. Ein Prozess, dessen Ende nicht in Sicht ist.

\section{Eulen nach Kassel}

Wie der Neoliberalismus in Athen wütet, wurde sicherlich allen Besucher_innen der documenta 14 irgendwann deutlich. Doch wozu in die Ferne schweifen? Auch in Kassel hätte man sich mit dem Neoliberalismus auseinandersetzen können. Doch das scheint nicht in das Weltbild der Kurator_innen zu passen, die wollten, dass in Kassel ein ,Echo“ ertönt. Das Bild vom Echo ist in der Tat eine gute Metapher für die Herangehensweise der Ausstellungsmacher_innen an den nordhessischen Veranstaltungsort. In den wenigen Statements des Leiters ist von Solidarität die Rede, doch davon spürte man in Kassel eigentlich wenig. Die lokalen Probleme, die der Logik der Weltwirtschaftsordnung folgen, waren ihnen unbekannt. Das betraf auch und gerade jenen Bereich, der den Kurator_innen besonders wichtig zu sein schien: die Flüchtlingsproblematik. Gleich zwei Objekte im öffentlichen Raum thematisierten sie. Das Röhrenprojekt des Iraker Hiwa K veranschaulichte das Leben von Flüchtlingen auf engstem Raum und unter schwierigsten Bedingungen. Dieses Projekt war in Zusammenarbeit mit Kasseler Studierenden und vor dem Hintergrund eigener Erfahrungen entstanden. Diese zu präsentieren ist nachvollziehbar. Warum aber kein Bezug

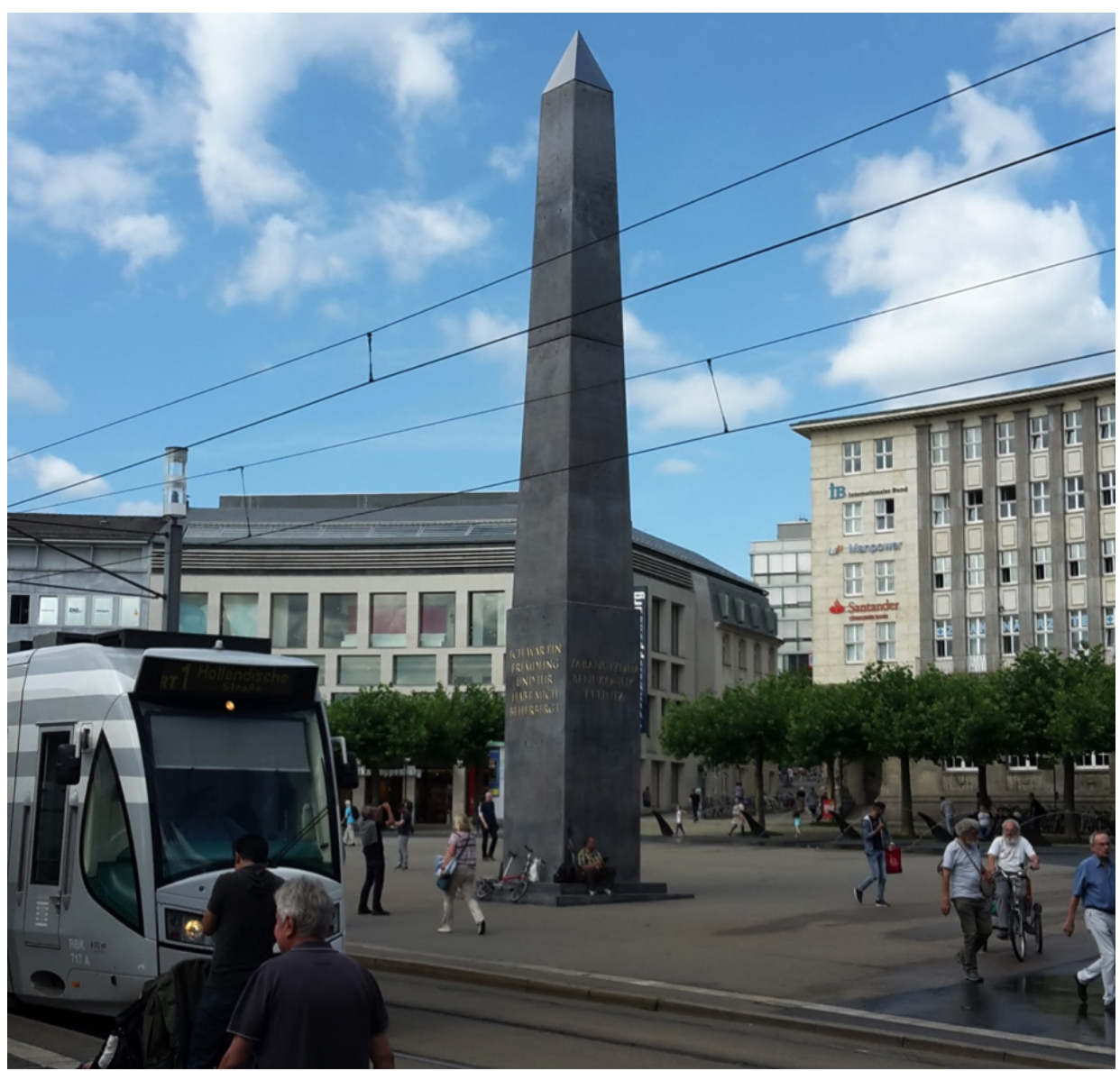


zum Flüchtlingslager in Kassel-Calden hergestellt wurde, bleibt unklar. Weit vor den Toren der Stadt wurde im Jahr 2015 zunächst eine Zeltstadt eingerichtet. Es kam zu Protesten, Bränden, Gewalt und Wachpersonal, das wegen Schießereien verurteilt wurde. Inzwischen sind von den 1.500 anvisierten Flüchtlingen nur noch ein Bruchteil dort, aber das Land hat sich entschieden, dieses Lager erheblich baulich zu verbessern und aufrechtzuerhalten für den nächsten ,Ansturm‘. Die Alternative wäre gewesen, dass man sich um mehr Wohnungen für Flüchtlinge in Kassel bemüht. Dazu müsste auf dem erheblich anziehenden Immobilienmarkt viel Geld in die Hand genommen werden. Lediglich acht Sozialwohnungen wurden im letzten Jahr in Kassel fertig gestellt. Mit viel Mühe wurde nun immerhin ein Plan für weitere 100 Sozialwohnungen in Helleböhn, einem sozialen Brennpunkt im Süden der Stadt, bewilligt. Wofür Land, Landkreis und Stadt aber sehr wohl große Summen zur Verfügung stellen, ist der neue Regionalflughafen Calden - nur ein Steinwurf von den Flüchtlingscontainern entfernt.

Wie lässt sich vor diesem Hintergrund das zweite Documenta-Monument zur Flüchtlingsproblematik - Olu Oguibes‘ Obelisk auf dem Königsplatz verstehen? Oguibe lobte damit die Gastfreundschaft und richtete sich gegen die global ausbreitende Anti-Flüchtlingspolitik. In dieser Globalität von Lob beziehungsweise Kritik ist wiederum schwierig auszumachen, warum es ausgerechnet in Kassel aufgestellt wurde und nicht in Washington, Warschau oder Budapest. Nach wie vor herrscht in Kassel eine relative Zustimmung und humanitäre Haltung in der Lokalpolitik und bei vielen verantwortlichen Akteur_innen gegenüber Flüchtlingen. Auch in der breiteren Stadtbevölkerung dürfte das noch der Fall sein. Jedoch sind die Alarmsignale ernst zu nehmen und die Probleme ins Auge zu fassen, die sich aus dem Zusammenleben mit den Flüchtlingen ergeben. Hierzu gehören einzelne Übergriffe auf Frauen, Kleinkriminalität und Alltagsrassismus. In Kassel haben zwar keine Neonazis das Sagen, hier brennen keine Heime. Das Engagement der Ehrenamtlichen hat dennoch abgenommen und viele professionelle und staatliche Institutionen operieren an ihren Grenzen. Mehr noch macht auch in Kassel der Terrorismus den Menschen Angst. Diese Angst hat durch lokale Vorkommnisse durchaus einen realen Kern. So hat der Staatsschutz eine islamistische Moschee geschlossen, weil der IS dort unter anderem erfolgreich Jugendliche für den Krieg in Syrien angeworben hatte.

„Ich frage mich oft, was die Menschen meinen, wenn sie sagen, Kassel habe sich in den letzten beiden Jahren - während der sogenannten Flüchtlingskrise - verändert“, meinte dazu Hendrik Folkerts vom Kurator_innenteam im Documenta-dagbook. „Liegt die Veränderung in der Angst, in den Appellen an populistische Fantasien, die rund um die Ankunft neuer Gemeinschaften große Ängste erzeugen, verkörpert durch Bewegungen wie die Alternative für Deutschland (AfD) mit ihrer extrem rechten Gesinnung?"[1] Die Antwort hat Kassel bei der Oberbürgermeister_innenwahl in diesem Jahr eindrucksvoll gegeben. Die AfD hatte nicht einmal eine Kandidatin oder einen Kandidaten aufgestellt. Linke, Grüne und SPD erhielten drei Viertel aller Stimmen. Obwohl die AfD hier Parteitage abhält, bekommt sie in der Stadt kein Bein auf den Boden. Das könnte man in Kassel alles relativ schnell wahrnehmen, wenn man sich denn auf die Stadtgesellschaft einlassen würde. 


\section{Echte Nazis}

Die Kommunikation mit Kassel wurde einem simplizistischen Weltbild geopfert, das einfach alles aus dem Wege ging, was in irgendeiner Weise das Narrativ vom globalen Unrecht irritieren könnte. Die documenta 14 betrieb im Sinne von Eric Hobsbawn eine, Wiederentdeckung von Geschichte (Hobsbawn/Ranger 2009) im geschützten Raum. Die Umwandlung des Fridericianums in einen öffentlichen Raum für das „Parlament der Körper“ war dafür das Paradebeispiel. Dieses wollte sich nicht auf die geschlossenen Räume beschränken und auch auf öffentlichen Plätzen agieren. Gemeint sind dabei vor allem die vielen Performances der documenta 14, die den Ort als Bühne für ihre Aufführungen auffassten. Die Besucher_innen oder Zuschauer_innen wurden als Teil eines, Chors' eingebunden. Wenig innovativ hatte man hierfür Lucius Burckhardts ,Spaziergänge‘ als Überbau-Vokabel verwendet, ohne den dialogischen und planungs- beziehungsweise interaktionsbezogenen Charakter der Spaziergangswissenschaften zu entfalten. Mit einer tatsächlichen Interaktion - gar mit der Konsequenz der Intervention - hatte diese Documenta nichts im Sinne. Es ging um Gesten, Auftritte, Vorführungen und vor allem Würdigung von Kämpfen gegen den globalen Kapitalismus - anderswo.

Das wurde insbesondere beim Thema Nationalsozialismus für Kassel zu einer fürchterlichen Erfahrung mit der documenta 14. In keiner Weise wurde auf die schwierige und nicht abgeschlossene Auseinandersetzung der Stadt mit dem Nationalsozialismus eingegangen. Das Hinweisschild auf die documenta 14 prangte am Rathaus direkt über dem Aschrott-Brunnen, der die tiefe Narbe verdeutlicht, die das Fehlen der jüdischen Bevölkerung in Kassel seit ihrer Deportation und Ermordung verdeutlicht. Ein eindringliches Bild im Zentrum der Stadt. An vielen anderen Plätzen wird ebenfalls aufgearbei-

Abb. 2 Das Parlament der Körper (Eigenes Foto) tet, wie der Faschismus funktioniert und gewirkt hat. Dabei handelt es sich um kleine Eingriffe in das Stadtbild, die aber die Omnipräsenz der totalitären Herrschaft besser verdeutlichen können als die Monumentalität der

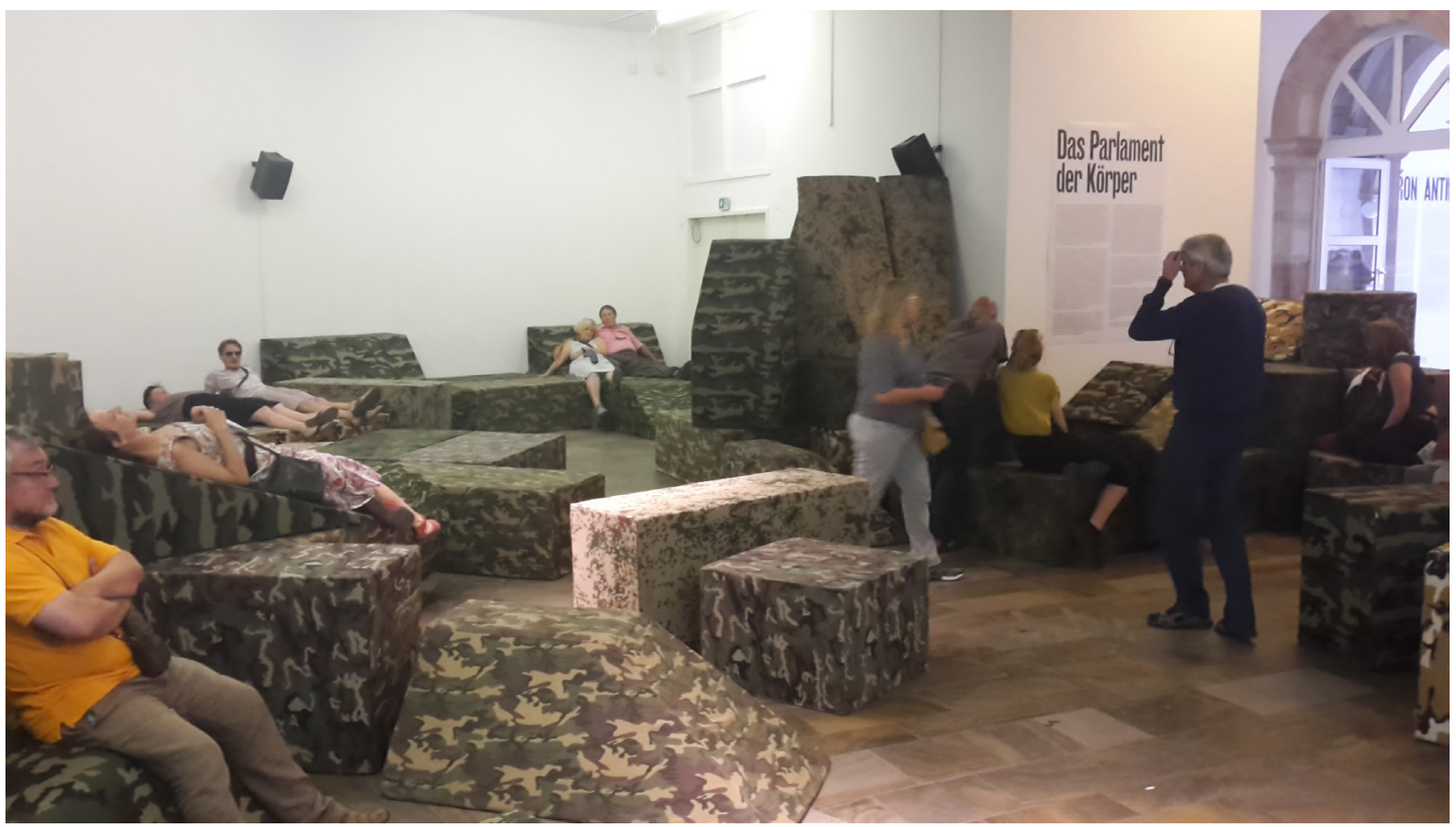




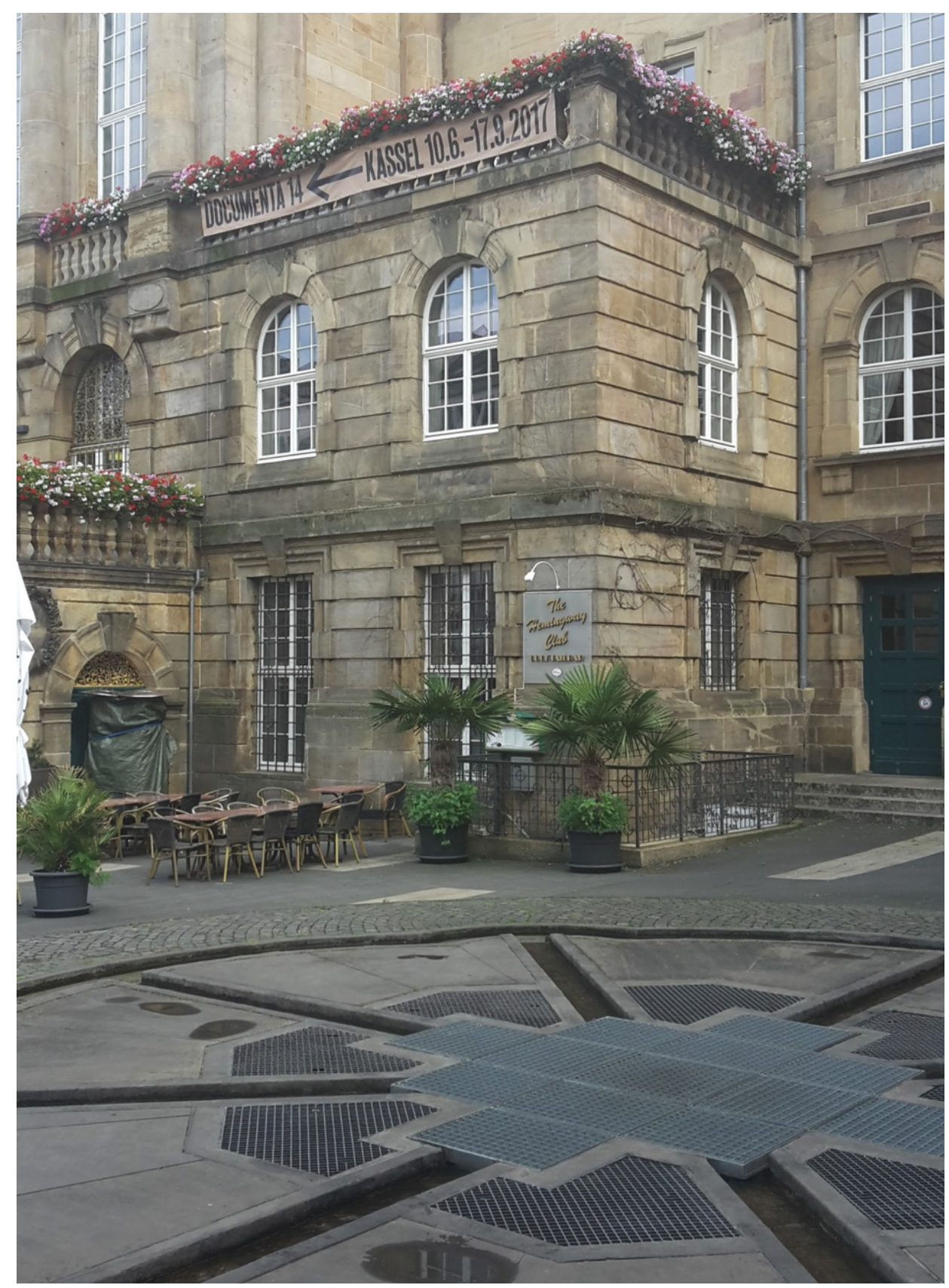

Abb. 3 Der Kasseler Aschrott-Brunnen (Eigenes Foto)

documenta 14. Diese stellte lieber Piotr Uklanskis Bilderschau mit 203 Fotos von vermeintlichen und wahren Nazis in der Neuen Galerie aus und vermittelte damit den Eindruck, der Nationalsozialismus sei vor allem eine personifizierte Herrschaft von bekannten Individuen gewesen. Wirklich auseinandersetzen wollte man sich mit der Frage, wie es zum Nationalsozialismus gekommen ist, sowieso nicht. Dieser erschien vielmehr nur als ein Glied in der langen Reihe der Ungerechtigkeiten vom Kolonialismus bis zum gestrigen Tag. Nicht bis heute, weil eine wirkliche tagesaktuelle Bezugnahme auf dieser Documenta ebenfalls fehlte: nichts über die Inhaftierung von Journalist_innen in der Türkei, nichts über den Populismus in Polen oder den USA, nichts über den Krieg im Jemen... Es ging um altes Unrecht, bei dem die Fronten zwischen ,Gut' und ,Böse“ im Nachhinein eindeutig sind.

Die Nutzung des Friedrichsplatzes - ein zentraler öffentlicher Ort - hätte sicherlich einen guten symbolischen Raum geboten, um ein Statement zu aktuellen Verfolgungen von Intellektuellen weltweit zu machen. Als Ort der Bücherverbrennung im Nationalsozialismus hätte hier der Spagat zwischen 
lokaler Bedeutung und globaler Message durchaus gelingen können. Mit Marta Minujíns „The Parthenon of Books“ war das ikonische Symbol der documenta 14 entstanden, das die Kasseler Bürger_innenschaft sicherlich beibehalten wird. In manchen Diskursen vor Ort wurde dieses Kunstwerk durchaus als eine Art Aktualisierung der Lehren aus dem Nationalsozialismus interpretiert. Umso enttäuschter konnte man sein, wenn man erfuhr, dass die dort eingeklebten Bücher jene waren, die während der argentinischen Militärdiktatur in den 1980er-Jahren verboten wurden. Bleibt der Parthenon als symbolische Repräsentanz der mit Athen verbundenen Originalität von Demokratie. Doch auch die war nicht gemeint. „Wir verzichten auf die gewohnte Gegenüberstellung von Diktatur und Demokratie, um so das Versagen der Demokratisierung unter dem Regime des Neoliberalismus besser zu verstehen“, heißt es im Begleittext zum Performance-Programm, den sogenannten „34 Freiheitsübungen“, die eine „ganz andere politische und poetische Landkarte“[2] zum Ziel haben.

\section{Ortslosigkeit als Konzept}

Die Kritik der Kunstwelt an dieser Documenta fiel mehr oder weniger einhellig negativ aus. Einer der Gründe für ihre mangelnde Überzeugungsfähigkeit lag in der provozierenden und wenig sinnlichen Art und Weise, dem Publikum etwas zu bieten, das auch einen emotionalen Bezugspunkt jenseits der Betroffenheit von Ungerechtigkeiten andernorts bietet. Der Kasseler Kunstwissenschaftler Harald Kimpel fasste die Ausstellung unter den Begriffen „radikale Entortung, Entzeitlichung und Entkunstung“ zusammen (vgl.

Abb. 4 Marta Minujíns „The Parthenon of Books" (Eigenes Foto)
Zeit Online, 30.07.2017). Diese Entortung hatte im Konzept von Adam Szymczyk einen grundsätzlichen Wert, der bei ihm mit weitergehenden normativen Vorstellungen verknüpft war. Dies wurde in der Ablehnung

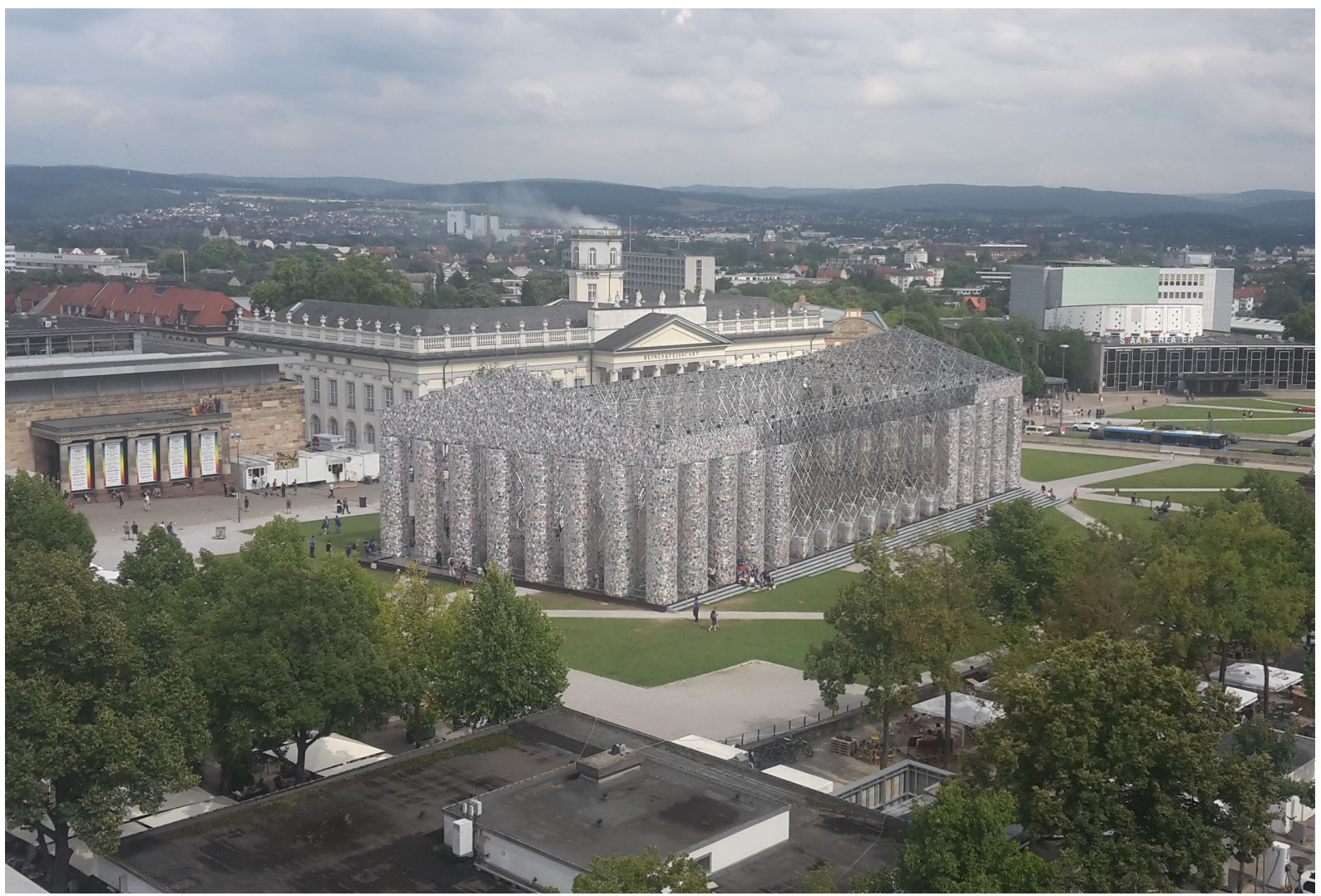


der beiden Vorgänger-Documentas eindringlich, denen er vorwarf, kein einheitliches Narrativ für die ,increasing diversity of artistic ideoms and formats“ (Szymczyk 2015: 239) zu konstruieren. Insbesondere wendete er sich gegen Carolyn Christov-Bakargievs Ansatz für die documenta 13, der es mit der Hinwendung zu Kassel nur um eine Stabilisierung des institutionellen Set-ups gegangen sei und die dabei die „revisionist ambitions“ (ebd.: 239) der Ausstellung fortgesetzt habe. Er hingegen forderte, dass die Documenta wieder einer „critical agency” (ebd.: 240) folge, ,instead of acting as witness, stage or prey of this spectacle" (ebd.). Deshalb habe man sich nicht auf Kassel beschränken dürfen und eine Reise nach Athen antreten müssen. Erstaunlicherweise wurde Athen sehr wohl als "physical, political location“ (ebd.: 243) betrachtet und die Aktionen des Programms ließen sich durchaus als Interventionen verstehen. Hierzu gehörte sicherlich vor allem der Versuch, dem „Nationalen Museum für Zeitgenössische Kunst“ (EMST) - auch durch die Ausstellung im Fridericianum - eine Perspektive zu eröffnen, mit der seine - bis dahin wegen der Finanzkrise in Athen nicht gezeigten - Ausstellungsstücke die Aufmerksamkeit der internationalen Kunstwelt erhielten, um vielleicht auf diese Weise dem Museum insgesamt eine $\mathrm{Zu}$ kunft zu ermöglichen. Das Künstlerkollektiv „Postcommodity“ widmete sich beispielsweise in Athen dem verwaisten Lyzeum des Aristoteles und brachte es mit dem früheren Offizierscasino durch akustische Installationen in Verbindung. Mit vielen öffentlichen Interaktionen, etwa auf dem zentralen Syntagma-Platz, erreichte die documenta 14 in Athen etwas, was sie in Kassel nicht anvisierte: Sie giff die Orte auf und trat mit ihnen in einen Dialog. Kassel verblieb als Stadt nur die Rolle als Bühne, als Schallwand für das Athener Echo. An dieser Ortslosigkeit, dem Monolog ohne Neugier, der sich nicht für die Widersprüche der eigenen Verortung öffnet, zerbrach diese Documenta.

\section{Endnoten}

[1] http://www.documenta14.de/de/artists/13500/mounira-al-solh (letzter Zugriff am 3.10.2017).

[2] http://www.documenta14.de/de/public-programs/928/34-freiheitsuebungen (letzter Zugriff am 12.09.2017).

\section{Autor_innen}

Frank Eckardt ist Politikwissenschaftler und Stadtsoziologe.

frank.eckardt@uni-weimar.de

\section{Literatur}

Hobsbawm, Eric / Ranger, Terence (Hg.) (2009): The invention of tradition. Cambridge [u.a.]: Cambridge Univ. Press.

Szymczyk, Adam (2015): documenta 14: Learning from Athens. In: Hans Eichel (Hg.), 60 Jahre documenta. Die lokale Geschichte einer Globalisierung. Berlin/Kassel: Siebenhaar, 237-248. 